\title{
Clubs and the Market: Large Finite Economies
}

\author{
Bryan Ellickson \\ Department of Economics \\ University of California, Los Angeles \\ Birgit Grodal \\ Institute of Economics \\ University of Copenhagen \\ Suzanne Scotchmer \\ Department of Economics, \\ Graduate School of Public Policy \\ University of California, Berkeley \\ William R. Zame \\ Department of Economics \\ University of California, Los Angeles
}

\author{
Working Paper Number 766 \\ Department of Economics \\ University of California, Los Angeles \\ Bunche 2263 \\ Los Angeles, CA 90095-1477 \\ April 9, 1997
}




\title{
Clubs and the Market: Large Finite Economies*
}

\author{
Bryan Ellickson \\ Department of Economics \\ University of California, Los Angeles \\ Birgit Grodal \\ Institute of Economics \\ University of Copenhagen \\ Suzanne Scotchmer \\ Department of Economics \\ and \\ Graduate School of Public Policy \\ University of California, Berkeley \\ William R. Zame \\ Department of Economics \\ University of California, Los Angeles
}

April 9, 1997

\footnotetext{
'We thank Robert Anderson for tutelage, Kenneth Arrow and Joe Ostroy for helpful arguments, and seminar audiences at CalTech, the Conference on City Formation at Washington University in St. Louis, the Midwest Mathematical Economics Meetings (Fall 1996), the Public Choice Society Meetings, the Santa Fe Institute, the Stanford Institute for Theoretical Economics (Summer 1996), UC Berkeley, UC Santa Cruz and UCLA, especially Mike Akemann, Larry Blume, Sara Castellanos, David Cooper, Mike Ryall and Federico Weinschelbaum, for comments. We thank the UCLA and UC Berkeley Academic Senate Committees on Research, the National Science Foundation and the Fullbright Foun-
} 


\begin{abstract}
This paper defines a general equilibrium model with exchange and club formation. Agents trade multiple private goods widely in the market, can belong to several clubs, and care about the characteristics of the other members of their clubs. The space of agents and the number of possible club types are finite. It is shown that (i) approximate competitive equilibrium exists, (ii) the core can approximately be decentralized. The approximations are close if the economy is large. The central subtlety is in modeling club memberships and expressing the notion that membership choices are consistent across the population.
\end{abstract}

JEL Classification Numbers D50, D51, D71

Keywords clubs, core convergence, approximate decentralization

dation for financial support and the UCLA and UC Berkeley Departments of Economics and the Institute of Economics of the University of Copenhagen for gracious hospitality (to Grodal, to Zame, and to Scotchmer and Zame, respectively) during preparation of this paper. 


\section{Introduction}

Consumption is typically a social activity. The company we keep affects our demand for private goods, and our consumption of private goods affects the company we seek. General equilibrium theory in the tradition of Arrow and Debreu focuses on the anonymous interactions of consumers with the market, largely ignoring the social aspect of consumption. Club theory in the tradition of Buchanan, on the other hand, focuses on the social activity of consumption, largely ignoring the anonymous interactions of individuals with the market. The principal purpose of this paper and our (1997) companion paper is to integrate club theory and general equilibrium theory, constructing a framework which incorporates widespread trading of private goods in competitive markets and individual consumption in small groups chosen voluntarily in equilibrium. This paper treats large finite economies; the companion paper treats continuum economies.

Following an idea of Buchanan (1965), we focus on clubs that are small relative to the economy: marriages, gyms, academic departments, firms, golfing foursomes, or the clienteles of restaurants. It seems natural to assume that each agent belongs to several such clubs simultaneously, and our model has this feature. We thus diverge from the previous club literature, which has assumed that each agent can belong to at most one club, and therefore that the club memberships form a partition of the population. ${ }^{1}$

We describe a (type of) club as a pair consisting of a description of the external characteristics of its members and a specified activity; thus we follow Ellickson (1979) and Mas-Colell (1981) as viewing the activity of a club as a public project rather than as provision of some level of a public good. A club membership is an opening in a club available to agents with specified

\footnotetext{
${ }^{1}$ This literature is vast. See for example Berglas (1976), Berglas and Pines (1981), Bewley (1981), Brueckner (1994), Conley and Wooders (1994), Gilles and Scotchmer (1997), Greenberg and Weber (1986), Greenberg and Shitovitz (1988) Ellickson (1973, 1979), Konishi (1996), Scotchmer (1985a, 1985b, 1994, 1996), Scotchmer and Wooders (1987a, 1987b), Wooders $(1978,1989)$.
} 
characteristics. Agents choose both private goods and club memberships, and private goods and club memberships are treated and priced in parallel fashion.

In most of the club literature (e.g., see Gilles and Scotchmer (1997) who studied replica economies), prices for memberships are constructed as willingness-to-pay prices after characterizing the decentralizing prices for private goods. This technique does not work if consumers can choose several club memberships. We treat club memberships and private goods in a unified manner, integrating private goods and memberships in a single commodity space.

Despite our unified treatment of private goods and club memberships, club economies differ in important ways from exchange economies. First, club memberships are indivisible. This indivisibility leads to an "integer problem" which is an obstacle both to the existence of equilibrium and to the decentralizability of core states. Because it seems to us that the indivisibility of club memberships is central to understanding clubs, we address it directly.

Second, club membership choices must be consistent across the population. If a third of the population are women married to men, for example, then a third of the population must be men married to women. Consistency must hold simultaneously for all types of clubs, and allow for the possibility that every individual may belong to several clubs.

Third, there is an important difference in the pricing of private goods and of club memberships: private good prices must be positive, but club membership prices may be positive, negative or zero.

We assume that the set of possible clubtypes is finite. Because our commodity space accommodates both private goods and club memberships, this assumption serves the technical purpose of leading to a finite dimensional commodity space. However finiteness of the set of possible clubtypes could be derived rather than assumed. The literature following Buchanan assumes that clubs have finite optimal sizes; hence if the economy is larger than these optimal sizes, there is only a finite collection that could possibly arise in 
equilibrium or in an efficient state of the economy. We restrict to this finite set at the outset. Of course not all the clubtypes in our finite set need be chosen. The clubtypes that are chosen in equilibrium will depend on the aggregate characteristics, and on consumers' preferences and endowments.

Our work builds on a long tradition in the club literature that seeks to demonstrate that clubs can arise as an endogenous outcome of competition in the market. The intuition is that if clubs are "small" relative to the market, then they have no market power.

In keeping with the view that perfect competition is best demonstrated in the continuum, our (1997) paper builds a continuum model. In that paper, we prove that equilibrium exists and that the core coincides with the set of competitive equilibrium states.

The continuum is convenient for applications, because it enables the analyst to ignore the "integer" problem, to treat the economy as competitive, and to assume that equilibrium exists. However, just as for exchange economies, the continuum would be of little interest if the results did not hold in an approximate sense for large finite economies. This paper extends approximation results for large finite exchange economies to club economies, using the same unrestrictive assumptions as in our paper on the continuum. We allow for general large finite economies, not just replicas; we make no convexity assumptions; and we permit each agent to belong to several clubs.

Our proof that the core can be approximately decentralized follows lines introduced by Anderson (1978), except that we must also accommodate the choices of club memberships and the necessity that these choices be consistent. This is accomplished through a combinatorial lemma which guarantees that if the club memberships chosen by a coalition are "almost" consistent, then the coalition contains a large subcoalition whose memberships are exactly consistent. The absolute (not proportional) difference in size between the coalition and the subcoalition is bounded.

In our notion of approximate equilibrium, the consumption of each agent is in his budget set, and most agents are optimizing. To construct an ap- 
proximate equilibrium, we first construct an "equilibrium" for an enlarged economy, and then, using the Shapley-Folkman Theorem and our combinatorial lemma, show that there is a feasible state for the original economy that is "almost" an equilibrium.

To cement the link between large finite economies and continuum economies, one must also know that the approximations become better as the economy grows, and converge quickly. Our theorems give rates of convergence comparable to those known for exchange economies. For the approximate decentralization theorem, the discrepancy between the core and a Walrasian equilibrium is measured in terms of consumers' budgets. We show that the per-capita budget discrepancy shrinks at rate $1 / n$, where $n$ is the size of the population. For existence of approximate equilibrium, the discrepancy from an equilibrium is measured by the number of agents who are not optimizing in their budget sets. This number is bounded by a constant, and in particular shrinks at rate $1 / n$ as a proportion of the population.

The description of the club economy is in Section 2, which also addresses the First Welfare Theorem. Section 3 presents our approximate decentralization result, and Section 4 presents our result on the existence of approximate equilibrium. Proofs are collected in Section 5. 


\section{Club Economies}

In this section we describe a club economy and define Pareto optimality, the core and equilibrium for such economies.

\subsection{Private Goods}

We assume throughout that there are $N \geq 1$ private goods, each perfectly divisible and publicly traded; the space of private goods is therefore $\mathbf{R}^{N}$. For $x, x^{\prime} \in \mathbf{R}^{N}$, we write $x \geq x^{\prime}$ to mean $x_{i} \geq x_{i}^{\prime}$ for each $i, x>x^{\prime}$ to mean that $x \geq x^{\prime}$ but $x \neq x^{\prime}$, and $x>>x^{\prime}$ to mean that $x_{i}>x_{i}^{\prime}$ for each $i$. We write $|x|=\sum_{n=1}^{N}\left|x_{n}\right|$.

\subsection{Clubs}

We describe a type of club by the number and characteristics of its members and the activity in which the club is engaged.

Formally, we let $\Omega$ be a finite set of external characteristics (of potential members of a club). An element $\omega \in \Omega$ is (or encodes) a complete description of the characteristics of an individual that are relevant for the other members of a club. For further discussion of the interpretation of external characteristics, see Section 2.10.

A profile is a function $\pi: \Omega \rightarrow \mathbf{Z}_{+}=\{0,1, \ldots\}$ describing the members of a club. For $\omega \in \Omega, \pi(\omega)$ represents the number of members of the club having external characteristic $\omega$. For $\pi$ a profile, write $|\pi|=\sum_{\omega \in \Omega} \pi(\omega)$ for the total number of members. We write 0 for the zero profile (representing the empty club).

The activities available to a profile of agents belong to a finite set $\Gamma$. We interpret the elements $\gamma \in \Gamma$ as public projects in the sense of Ellickson (1979) and Mas-Colell (1980), rather than as public goods in the sense of 
Samuelson. Activities are not traded.

A club type is a pair $c=(\pi, \gamma)$ consisting of a profile and an activity. We take as given a finite set of possible club types Clubs $=\{(\pi, \gamma)\}$. We find it convenient to treat singleton clubs separately, so we assume that $|\pi| \geq 2$ for all $(\pi, \gamma) \in$ Clubs. $^{2}$ Formation of the club $(\pi, \gamma)$ requires a total input of private goods equal to $\operatorname{inp}(\pi, \gamma) \in \mathbf{R}_{+}^{N}{ }^{3}$

A club membership is an opening in a particular type of club for an agent of a particular external characteristic; i.e., a triple $m=(\omega, \pi, \gamma)$ such that $(\pi, \gamma) \in$ Clubs and $\pi(\omega) \geq 1$. (An agent can belong to a club only if the description of that club type includes one or more members of his/her external characteristics.) Write $\mathcal{M}$ for the set of club memberships.

Each agent may choose to belong to many clubs or to none. A list is a function $\ell: \mathcal{M} \rightarrow\{0,1, \ldots\} ; \ell(\omega, \pi, \gamma)$ specifies the number of memberships of type $(\omega, \pi, \gamma)$ chosen by an agent. Write:

$$
\text { Lists }=\{\ell: \ell \text { is a list }\}
$$

for the set of lists. We frequently find it convenient to view Lists (which is a set of functions from $\mathcal{M}$ to $\{0,1, \ldots\}$ ) as a subset of $\mathbf{R}^{\mathcal{M}}$ (which is the set of functions from $\mathcal{M}$ to $\mathbf{R}$ ).

\subsection{Agents}

A complete description of an agent $a \in A$ consists of his/her external characteristics, choice set, endowment of private goods and utility function. ${ }^{4}$ An external characteristic is an element $\omega_{a} \in \Omega$. The choice set $X_{a}$ for an agent

\footnotetext{
${ }^{2}$ Since activities are not traded, the choice of activities of singleton clubs can be incorporated into preferences.

${ }^{3}$ More generally, we could assume that each project could be produced from any input vector from some specified set and incorporate the choice of production technology into our notion of feasibility.

${ }^{4}$ We use utility functions rather than preferences as a matter of convenience; under the assumptions made here, the two specifications are equivalent.
} 
$a \in A$ specifies which bundles of private goods and which choices of club memberships are feasible, so $X_{a} \subset \mathrm{R}^{N} \times$ Lists. For simplicity, we assume that the only restriction on private good consumption is that it be nonnegative, so that $X_{a}=\mathbf{R}_{+}^{N} \times \operatorname{Lists}(a)$ for some subset $\operatorname{Lists}(a) \subset \operatorname{Lists}$; we assume $0 \in \operatorname{Lists}(a)$ for each $a$, so club formation is not necessary for survival. ${ }^{5}$ We assume that $\ell(\omega, \pi, \gamma)=0$ for every $(\omega, \pi, \gamma) \in \mathcal{M}$ for which $\omega \neq \omega_{a}$; that is, no individual may choose membership in any club type containing no members of his/her external characteristic. We also assume throughout that there is an exogenously given upper bound $M$ on the number of memberships an individual may choose, so $|\ell| \leq M$ for each $\ell \in \operatorname{Lists}(a)$. The utility function for agent $a$ is defined over private goods consumptions and club memberships and is thus a mapping $u_{a}: X_{a} \rightarrow \mathbf{R}$.

We assume throughout that utility functions are strictly monotone in private goods; i.e., $u_{a}(x, \ell)>u_{a}\left(x^{\prime}, \ell\right)$ for $a \in A, x, x^{\prime} \in \mathbf{R}_{+}^{N}, x>x^{\prime}$. However, we make no assumption that utility is monotone in the level of any activity; indeed, in our framework it is meaningless to talk about the level of an activity. The ranking of activities may be different for different individuals, and an individual's ranking of activities may depend on his/her consumption of private goods. ${ }^{6}$ We take the view that an agent's preferences for private goods and for club memberships are interdependent and cannot be disentangled (except for monotonicity in private goods).

\subsection{Club Economies}

A club economy $\mathcal{E}$ is a finite set $A$ of agents and a mapping $a \mapsto\left(\omega_{a}, X_{a}, e_{a}, u_{a}\right)$ that assigns to each agent $a \in A$ his external characteristic, choice set, endowment and utility function. As above, we assume that utility functions are continuous and strictly monotone in private goods.

\footnotetext{
${ }^{5}$ Thus we incorporate into consumption sets various kinds of restrictions on club memberships. For instance, we may forbid membership in 2 marriages. More general specifications of consumption sets would be easily accommodated.

${ }^{6}$ See Diamantaras and Gilles (1996), Gilles and Scotchmer (1997) and Diamantaras, Gilles and Scotchmer (1996) for further discussion on this point.
} 
We assume that the aggregate endowment

$$
\bar{e}=\sum_{a \in A} e_{a}
$$

is strictly positive, so all private goods are represented in the aggregate.

\subsection{States}

A state of a club economy is a mapping

$$
f=(x, \mu): A \rightarrow \mathbf{R}^{N} \times \mathbf{R}^{\mathcal{M}}
$$

A state describes choices for each individual agent, ignoring feasibility at the level of the individual and at the level of society. Individual feasibility means that $\left(x_{a}, \mu_{a}\right) \in X_{a}$. Social feasibility entails market clearing for private goods and consistent matching of agents.

We define a property of choice functions $\mu: B \rightarrow$ Lists and show that it is equivalent to a property of aggregate membership vectors $\sum_{B} \mu_{a}$. For each integer $j>0$, let

$$
E_{\mu}^{j}(\omega, \pi, \gamma)=\left\{a \in B: \mu_{a}(\omega, \pi, \gamma)>0\right\}
$$

This is the set of agents who choose $j$ memberships of type $(\omega, \pi, \gamma)$. Write $|E|$ for the number of agents in $E$, so that $\left|E_{\mu}^{j}(\omega, \pi, \gamma)\right|$ is the number of agents who choose $j$ memberships of type $(\omega, \pi, \gamma)$ and $j\left|E_{\mu}^{j}(\omega, \pi, \gamma)\right|$ is the number of memberships of type $(\omega, \pi, \gamma)$ chosen by these agents. The sum

$$
\sum_{j=1}^{\infty} j\left|E_{\mu}^{j}(\omega, \pi, \gamma)\right|
$$

is thus the total number of memberships of type $(\omega, \pi, \gamma)$ chosen by all agents. We say that a function $\mu: B \rightarrow$ Lists is integer consistent for $B$ if for each $(\pi, \gamma) \in \mathcal{M}$ there is a non-negative integer $\alpha(\pi, \gamma)$ such that

$$
\sum_{j=1}^{\infty} j\left|E_{\mu}^{j}(\omega, \pi, \gamma)\right|=\alpha(\pi, \gamma) \pi(\omega)
$$


for every $\omega \in \Omega{ }^{7}$

We say that a club membership vector $\bar{\mu} \in \mathbf{R}^{\mathcal{M}}$ is integer consistent if for every club type $(\pi, \gamma)$ there is a nonnegative integer $\alpha(\pi, \gamma)$ such that

$$
\bar{\mu}(\omega, \pi, \gamma)=\alpha(\pi, \gamma) \pi(\omega)
$$

for every $\omega \in \Omega$. Write

$$
\text { Cons }^{*}=\left\{\bar{\mu} \in \mathbf{R}^{\mathcal{M}}: \bar{\mu} \text { is integer consistent }\right\}
$$

Write Cons $\subset \mathbf{R}^{\mathcal{M}}$ for the linear subspace of $\mathbf{R}^{\mathcal{M}}$ spanned by Cons ${ }^{*}$.

The following lemma, whose simple proof is left to the reader, states the relationship between the two notions of integer consistency.

Lemma 2.1 Let $\mathcal{E}$ be a club economy, let $B \subset A$ be a nonempty subset, and let $\mu: B \rightarrow$ Lists be a function. Then $\mu$ is integer consistent for $B$ if and only if $\sum_{a \in B} \mu_{a} \in$ Cons $^{*}$.

We say that the state $f=(x, \mu)$ is feasible for the subset $B \subset A$ if it satisfies the following requirements:

(i) Individual Feasibility

$$
\left(x_{a}, \mu_{a}\right) \in X_{a} \text { for each } a \in A
$$

(ii) Material Balance ${ }^{8}$

$$
\sum_{a \in B} x_{a}+\sum_{a \in B} \sum_{(\omega, \pi, \gamma) \in \mathcal{M}} \frac{1}{|\pi|} \operatorname{inp}(\pi, \gamma) \mu_{a}(\omega, \pi, \gamma)=\sum_{a \in B} e_{a}
$$

\footnotetext{
${ }^{7}$ We use the term "integer consistent" to distinguish the present notion from the related, but different, notion, termed "consistent," in our companion (1997) paper.

${ }^{8}$ Material balance means that the social consumption of private goods (within $B$ ) plus the quantity of private goods used as inputs to club activities (by members of $B$ ) is equal to the social endowment of private goods (within $B$ ).
} 


\section{(iii) Integer Consistency}

$\mu$ is integer consistent for $B$

We say the state $f$ is feasible if it is feasible for the set $A$ itself.

Our description of feasible states of the economy is different from the description of feasible states in most of club theory, where the analog of integer consistency is expressed by a requirement that clubs form a partitition of the set of agents. Our description allows for the possibility that agents belong to many clubs, that different agents belong to different numbers of clubs, and that clubs have overlapping memberships. For instance, agents may be married, have employment in a firm, belong to a gym, attend movies and concerts, take meals in a restaurant, and so forth. In the special case that agents can belong to only one club $(M=1)$, integer consistency reduces to the assertion that clubs form a partition.

We do not keep track of which person belongs to which club, nor do we need to do so: every function $\mu: A \rightarrow$ Lists assigning memberships to agents that is integer consistent for $A$ corresponds to an integer consistent membership vector (and vice versa). Of course, a given membership vector may correspond to many assignments of memberships to agents, but we do not need to distinguish them, because we assume that individuals care only about the external characteristics of their consumption partners, not about their identities. (See Section 2.10.)

\subsection{Pareto Optimality and the Core}

As in the exchange setting, we distinguish two notions of Pareto optimality and the core; the stronger notion allows blocking if some agents (in the relevant group) are made better off and none are made worse off, the weaker notion requires that all agents be made better off. For exchange economies, strict monotonicity of preferences guarantees that the two notions coincide. Because choices of club memberships are indivisible, however, the notions 
may be distinct, even if preferences are strictly monotone in private goods. In this subsection we define two notions of Pareto optimality and the core and give a natural condition that guarantees that they coincide.

Let $f$ be a feasible state. We say that $f$ is weakly Pareto optimal if there is no feasible state $g$ such that $u_{a}(g(a))>u_{a}(f(a))$ for all $a \in A ; f$ is strongly Pareto optimal if there is no feasible state $h$ such that $u_{a}(h(a)) \geq u_{a}(f(a))$ for all $a \in A$ and $u_{a^{\prime}}(h(a))>u_{a^{\prime}}(f(a))$ for all $a$ in some nonempty subset $A^{\prime} \subset A$. Note that strong Pareto optimality is a more restrictive notion than weak Pareto optimality. Similarly, $f$ is in the weak core if there is no nonempty subset $B \subset A$ state $g$ that is feasible for $B$ such that $u_{b}(g(b))>u_{b}(f(b))$ for every $b \in B ; f$ is in the strong core if there is no subset nonempty $B \subset A$ and state $h$ that is feasible for $B$ such that $u_{b}(h(b)) \geq u_{b}(f(b))$ for every $b \in B$ and $u_{b^{\prime}}\left(h\left(b^{\prime}\right)\right)>u_{b^{\prime}}\left(f\left(b^{\prime}\right)\right)$ for all $b^{\prime}$ in some nonempty subset $B^{\prime} \subset B$. The strong core is a subset of the weak core.

In general, weakly Pareto optimal allocations may not be strongly Pareto optimal, and the weak core may be a proper superset of the strong core. The following assumption, adapted from Gilles and Scotchmer (1997), guarantees that weak and strong Pareto optimality coincide and that the weak and strong cores coincide.

We say that endowments are desirable if for every agent $a$ and every list $\ell \in \operatorname{Lists}(a), u_{a}\left(e_{a}, 0\right)>u_{a}(0, \ell)$. That is, each agent would prefer to remain single and consume his endowment rather than to belong to any feasible set of clubs and consume no private goods. Desirability of endowments is weaker than the assumption Mas-Colell (1980) refers to as essentiality of private goods, which in our framework would be:

$$
u_{a}(0, \ell)=\min _{\left(x^{*}, \ell^{*}\right) \in X_{a}} u_{a}\left(x^{*}, \ell^{*}\right)
$$

for every $\ell \in \operatorname{Lists}(a)$. We omit the straightforward proof of the following proposition. $^{9}$

Proposition 2.2 If endowments are desirable, then weak and strong Pareto

\footnotetext{
${ }^{9}$ For details, see our companion paper (1997).
} 
optimality coincide and the weak and strong core coincide.

When endowments are desirable, we omit the modifiers and refer unambiguously to Pareto optimality and the core.

\subsection{Equilibrium}

Our notion of equilibrium involves the pricing of private goods and of club memberships. Private goods prices $p$ lie in $\mathbf{R}^{N}$; prices for club memberships $q$ lie in $\mathbf{R}^{\mathcal{M}}$, so the vector of all prices lies in $\mathbf{R}^{N} \times \mathbf{R}^{\mathcal{M}}$. Because we assume that preferences are monotone in private goods, we will require that private goods prices be non-negative. However, prices for club memberships may be positive, negative or zero; prices for club memberships include transfers between agents in a given club - some agents may subsidize others. For $(x, \bar{\mu}) \in \mathbf{R}^{N} \times \mathbf{R}^{\mathcal{M}}$ a vector of private goods and club memberships and $(p, q) \in \mathbf{R}^{N} \times \mathbf{R}^{\mathcal{M}}$ a vector of prices, write

$$
(p, q) \cdot(\bar{x}, \bar{\mu})=p \cdot \bar{x}+q \cdot \bar{\mu}
$$

for the cost of $(\bar{x}, \bar{\mu})$.

A club equilibrium consists of a feasible state $f=(x, \mu)$, private good prices $p \in \mathbf{R}_{+}^{N} \backslash\{0\}$ and club membership prices $q \in \mathbf{R}^{\mathcal{M}}$, satisfying the conditions:

(1) Budget Feasibility for Individuals

For all $a \in A$ :

$$
p \cdot x_{a}+q \cdot \mu_{a} \leq p \cdot e_{a}
$$

(2) Optimization

For all $a \in A$ :

$$
\left(x_{a}^{\prime}, \mu_{a}^{\prime}\right) \in X_{a} \text { and } u_{a}\left(x_{a}^{\prime}, \mu_{a}^{\prime}\right)>u_{a}\left(x_{a}, \mu_{a}\right) \Rightarrow p \cdot x_{a}^{\prime}+q \cdot \mu_{a}^{\prime}>p \cdot e_{a}
$$




\section{(3) Budget Balance for Clubs}

For each club type $(\pi, \gamma) \in$ Clubs:

$$
\sum_{\omega \in \Omega} \pi(\omega) q(\omega, \pi, \gamma)=p \cdot \operatorname{inp}(\pi, \gamma)
$$

Thus, at an equilibrium, individuals optimize subject to their budget constraint and the total cost of memberships in a given club is just enough to pay for the inputs to the given activity.

\subsection{Pure Transfers}

Our formulation of equilibrium requires that the sum of membership prices in each club type be exactly sufficient to pay for the inputs required for production of the club activity. An equivalent notion makes clear the role of membership prices as taxes and subsidies (and will prove to be more convenient in proofs).

Say that $q \in \mathbf{R}^{\mathcal{M}}$ is a pure transfer if $q \in$ Trans, defined as:

$$
\text { Trans }=\left\{q \in \mathbf{R}^{\mathcal{M}}: q \cdot \mu=0 \text { for each } \mu \in \text { Cons }\right\}
$$

Thus for each club type $(\pi, \gamma)$ and $q \in$ Trans,

$$
\sum_{\omega \in \Omega} \pi(\omega) q(\omega, \pi, \gamma)=0
$$

A pure transfer equilibrium is a triple $(f, p, q)$ where $f$ is a feasible state, $p \in \mathbf{R}_{+}^{N} \backslash\{0\}$ is a vector of private good prices and $q \in \mathbf{R}^{\mathcal{M}}$ is a vector of membership prices satisfying the conditions:

\section{(1) Budget Feasibility}

For almost all $a \in A$,

$$
p \cdot x_{a}+q \cdot \mu_{a}+\sum_{(\omega, \pi, \gamma)} p \cdot \frac{1}{|\pi|} \operatorname{inp}(\pi, \gamma) \mu_{a}(\omega, \pi, \gamma) \leq p \cdot e_{a}
$$




\section{(2) Optimization}

For almost all $a \in A$, if $\left(x_{a}^{\prime}, \mu_{a}^{\prime}\right) \in X_{a}$ and

$$
u_{a}\left(x_{a}^{\prime}, \mu_{a}^{\prime}\right)>u_{a}\left(x_{a}, \mu_{a}\right)
$$

then

$$
p \cdot x_{a}^{\prime}+q \cdot \mu_{a}^{\prime}+\sum_{(\omega, \pi, \gamma)} p \cdot \frac{1}{|\pi|} \operatorname{inp}(\pi, \gamma) \mu_{a}^{\prime}(\omega, \pi, \gamma)>p \cdot e_{a}
$$

(3) Pure Transfers

$$
q \in \text { Trans }
$$

The following lemma tells us that equilibrium and pure transfer equilibrium are equivalent notions; we leave the simple proof to the reader.

Lemma 2.3 Let $\mathcal{E}$ be a club economy. For $q^{*} \in$ Trans define $q \in \mathbf{R}^{\mathcal{M}}$ by

$$
q^{*}(\omega, \pi, \gamma)=q(\omega, \pi, \gamma)+p \cdot \frac{1}{|\pi|} \operatorname{inp}(\pi, \gamma)
$$

Then: $(f, p, q)$ is a pure transfer equilibrium if and only if $\left(f, p, q^{*}\right)$ is an equilibrium.

\subsection{The First Welfare Theorem and the Core}

In our club context, as in the exchange case, we easily obtain the first welfare theorem. We omit the straightforward proof. ${ }^{10}$

Theorem 2.4 Every equilibrium state of a club economy belongs to the weak core, and in particular is weakly Pareto optimal. If endowments are desirable, every equilibrium state belongs to the strong core, and in particular is strongly Pareto optimal.

\footnotetext{
${ }^{10}$ For details, we again refer to our companion paper (1997).
} 


\subsection{Discussion}

In our model, agents care about their own consumption and about the external characteristics of others in their clubs. The characteristics we have in mind should be observable to others in the club, which is why we call them external. Such characteristics might include sex, intelligence, appearance, even tastes and endowments, to the extent that such characteristics can be observed by others. ${ }^{11}$ On the other hand, we exclude private characteristics which are known only to the individual. Because we assume that memberships are priced according to external characteristics, our construction can be viewed as a compromise between the non-discriminatory pricing of competitive equilibrium and the personalized prices of Lindahl. To capture the essence of club theory, we regard as essential a certain degree of anonymity, but we also think it important to recognize that clubs offer different types of membership. ${ }^{12}$

One restriction in this model, which would be particularly desirable to eliminate in future work is that external characteristics are ascriptive, not acquired. Intelligence and endowments (if observable) are possible external characteristics, skill and consumption are not.

Of course we could formulate a model in which preferences for club memberships depend on various characteristics of club partners, but insist that prices be independent of those characteristics. In that case, however, and in contrast to the results proved here, core allocations might not be decentralizable by prices, and equilibria could fail to exist. (A similar comment

\footnotetext{
${ }^{11}$ But keep in mind that we assume in this paper that the set of external characteristics is finite.

${ }^{12}$ Much of the club literature indexes both the external characteristics and the tastes and endowments by a single "type;" see Berglas (1976), Gilles and Scotchmer (1997) for instance. Our use of external characteristics is closer in spirit to Conley and Wooders (1994), Engl and Scotchmer (1996) and Scotchmer (1996), where prices are understood as "externality prices." However, these latter papers treat only finite TU economies with a single private good, restrict agents to belong to at most one club, and do not discuss existence.
} 
applies to the possibility of preferences that depend on the consumptions of club partners.) 


\section{Approximate Decentralization}

In this section we extend Anderson's (1978) elementary core equivalence theorem to the club context, showing that for large finite club economies, core states can be approximately supported by prices. Because of the integer consistency requirement for blocking coalitions, the extension is not straightforward.

Following Anderson, we define two measures of how well a given price system approximately decentralizes a feasible state. Let $\mathcal{E}$ be a finite club economy, let $f=(x, \mu)$ be a feasible state, let $p \in \Delta=\left\{p \in \mathbf{R}_{+}^{N}: \sum p_{i}=1\right\}$ be a normalized system of private goods prices, and let $q \in \mathbf{R}^{\mathcal{M}}$ be a club membership price. For $r \in \mathbf{R}$ we write

$$
r^{+}=\max \{r, 0\}
$$

for the positive part of $r$. For $a \in A$ we define

$$
\begin{aligned}
& \rho_{a}^{1}(f, p, q)=\left[(p, q) \cdot\left(x_{a}, \mu_{a}\right)-p \cdot e_{a}\right]^{+} \\
& \rho_{a}^{2}(f, p, q)=\max \left\{\left[p \cdot e_{a}-(p, q) \cdot\left(x^{\prime}, \mu^{\prime}\right)\right]^{+}: u_{a}\left(x^{\prime}, \mu^{\prime}\right)>u_{a}\left(x_{a}, \mu_{a}\right)\right\}
\end{aligned}
$$

The number $\rho_{a}^{1}(f, p, q)$ measures how far agent $\left(x_{a}, \mu_{a}\right)$ lies outside agent $a$ 's budget set. The number $\rho_{a}^{2}(f, p, q)$ measures how much agent $a$ can save and still choose something preferred to $\left(x_{a}, \mu_{a}\right)$. Note that $\rho_{a}^{1}(f, p, q)=0$ if and only if $\left(x_{a}, \mu_{a}\right)$ lies in agent $a$ 's budget set and that $\rho_{a}^{2}(f, p, q)=0$ if and only if nothing preferred to $\left(x_{a}, \mu_{a}\right)$ is strictly cheaper. Define:

$$
\begin{aligned}
& \rho^{1}(f, p, q)=\frac{1}{|A|} \sum_{a \in A} \rho_{a}^{1}(f, p, q) \\
& \rho^{2}(f, p, q)=\frac{1}{|A|} \sum_{a \in A} \rho_{a}^{2}(f, p, q)
\end{aligned}
$$

The number $\rho^{1}(f, p, q)$ is a measure of the average deviation from individual budget sets, and the number $\rho^{2}(f, p, q)$ is a measure of the average deviation from individual optimization. 
In what follows we fix the set $\Omega$ of external characteristics, the set Clubs of possible club types, the bound $M$ on the number of memberships that may be chosen by any individual, and the number $N$ of private goods. Write $\operatorname{Econ}(\Omega$, Clubs, $M, N)$ for the set of finite club economies sharing this data.

Our approximate decentralization result is the following:

Theorem 3.1 There is a constant $K$ depending only on $\Omega$, Clubs, $M, N$ such that:

If $\mathcal{E} \in \operatorname{Econ}(\Omega$, Clubs, $M, N)$ is a finite club economy with $|A|>$ $K$ and $f=(x, \mu)$ is a core state then there is a normalized price system $(p, q) \in \Delta \times \mathbf{R}^{\mathcal{M}}$ such that

$$
\begin{aligned}
& \rho^{1}(f, p, q) \leq \frac{K}{|A|} \max \left\{\left|e_{a n}\right|: a \in A, 1 \leq n \leq N\right\} \\
& \rho^{2}(f, p, q) \leq \frac{K}{|A|} \max \left\{\left|e_{a n}\right|: a \in A, 1 \leq n \leq N\right\}
\end{aligned}
$$

and

$$
\sum_{\omega \in \Omega} \pi(\omega) q(\omega, \pi, \gamma)=p \cdot \operatorname{inp}(\pi, \gamma) \text { for each }(\pi, \gamma) \in \text { Clubs }
$$

(Note that the budgets of club types balance exactly.)

As in Anderson (1978), the proof constructs an approximately decentralizing price by separating the convex hull of the aggregate net preferred set from a translate of an appropriate cone. There are three subtleties:

(i) In Anderson (1978), the cone from which the aggregate preferred set is separated is the cone of feasible aggregate net trades, which is the negative orthant. In our context, the set of feasible aggregate net trades is the product of the negative orthant with the set Cons* of integer consistent membership choices, but this product is not a convex cone. The convex cone generated by the set of feasible aggregate net trades is the product of the negative orthant with the subspace Cons. 
(ii) A hyperplane that separates the aggregate net preferred set from a translate of the convex cone generated by the set of feasible aggregate net trades will yield prices $(p, q) \neq 0$, but it need not be the case that $p \neq 0$. To ensure $p \neq 0$ we separate from a translate of a slightly larger cone.

(iii) To show that the convex hull of the net aggregate preferred set is disjoint from our cone, we will need to show that, if it were not, we would be able to construct a blocking coalition. In constructing this blocking coalition, we must be sure that both private good consumptions and membership choices are feasible. Feasibility of private good consumption will be accomplished by "throwing out" a few agents. When we do this, however, we may find that the membership choices of the remaining agents are no longer integer consistent. The lemmas below show that we can restore integer consistency of membership choices by "throwing out" still more agents. This is a subtle and difficult problem because we must deal head on with the indivisibility and multiplicity of club membership choices.

We first isolate an abstract idea and result. Write $\mathbf{Z}_{+}^{n} \subset \mathbf{R}^{n}$ for the subset consisting of vectors whose coordinates are non-negative integers. We say that a non-empty subset $\mathcal{H} \subset \mathbf{Z}_{+}^{n}$ is closed under addition and relative subtraction if:

(i) $x, x^{\prime} \in \mathcal{H} \Rightarrow x+x^{\prime} \in \mathcal{H}$

(ii) $x, x^{\prime} \in \mathcal{H}, x-x^{\prime} \geq 0 \Rightarrow x-x^{\prime} \in \mathcal{H}$

We say that $\mathcal{G} \subset \mathcal{H}$ generates $\mathcal{H}$ if every element of $\mathcal{H}$ can be written as a non-negative integer combination of elements of $\mathcal{G}$; that is, for every $x \in \mathcal{H}$ there are non-negative integers $n_{x}(y), y \in \mathcal{G}$ with

$$
x=\sum_{y \in \mathcal{G}} n_{x}(y) y
$$


Lemma 3.2 Every non-empty subset $\mathcal{H} \subset \mathrm{Z}_{+}^{n}$ which is closed under addition and relative subtraction is generated by a finite set.

Note that Cons ${ }^{*} \subset \mathbf{Z}_{+}^{\mathcal{M}}$ is closed under addition and relative subtraction; let $\mathcal{G}_{1}$ be a finite set of generators.

Write

$$
\text { Lists }_{M}=\{\ell \in \text { Lists : }|\ell| \leq M\}
$$

We view Lists $_{M}$ as the unit vectors in $\mathbf{R}^{\text {Lists }_{M}}$, and define a linear map $T: \mathbf{R}^{\text {Lists }_{M}} \rightarrow \mathbf{R}^{\mathcal{M}}$ by $T\left(\delta_{\ell}\right)=\ell$ for each unit vector $\delta_{\ell}$. Set

$$
\mathcal{J}=\left\{x \in \mathbf{Z}_{+}^{\text {Lists }_{M}}: T(x) \in \text { Cons }^{*}\right\}
$$

The set $\mathcal{J}$ describes integer consistency in $\mathbf{R}^{\text {Lists }_{M}}$. It is easily checked that $\mathcal{J}$ is closed under addition and relative subtraction; let $\mathcal{G}_{2}$ be a finite set of generators.

Define constants $K_{1}, K_{2}$ by:

$$
\begin{aligned}
K_{1} & =2\left(\max _{g \in \mathcal{G}_{1}}|g|+1\right)\left(\max _{g^{\prime} \in \mathcal{G}_{2}}\left|g^{\prime}\right|+1\right) \\
K_{2} & =K_{1}|\mathcal{M}|
\end{aligned}
$$

Note that $K_{1}, K_{2}$ depend only on the set $\Omega$ of external characteristics, on the set Clubs of possible club types, and on the bound $M$ on the number of memberships that may be chosen by an individual. As we shall see, the constant in the statement of Theorem 3.1 is

$$
K=K_{1}\left(M N+M\left|\operatorname{Lists}_{M}\right|+1\right)+K_{2}+N+\left|\operatorname{Lists}_{M}\right|
$$

The following lemma will allow us to "throw out" the right set of agents.

Lemma 3.3 If $B$ is a finite set and $\nu: B \rightarrow \operatorname{Lists}_{M}$ is a function then there is a subset $B^{\prime} \subset B$ such that

$$
\sum_{b \in B^{\prime}} \nu_{b} \in \text { Cons }^{*}
$$


and

$$
\left|B \backslash B^{\prime}\right| \leq K_{1} \operatorname{dist}\left(\sum_{b \in B} \nu_{b}, \text { Cons }\right)+K_{2}
$$




\section{Approximate Equilibrium}

It is not obvious what the "right" notion of approximate equilibrium should be. Our approximate decentralization result Theorem 3.1 shows that core states are approximate equilibria in the sense that, on average, agents are nearly in their budget sets and nearly optimize in their budgets. In this section we establish the existence of feasible states satisfying a stronger approximate equilibrium notion: all agents are in their budget sets and most agents exactly optimize in their budget sets. (In both cases we require that budgets of club types exactly balance.)

As in Section 3, we fix $\Omega$, Clubs, $M, N$. Write

$$
M^{*}=\max \{|\pi(\omega)|:(\pi, \gamma) \in \text { Clubs, } \omega \in \Omega\}
$$

$K_{1}, K_{2}$ are the constants constructed at the end of Section 3.

Theorem 4.1 If $\mathcal{E} \in \operatorname{Econ}(\Omega$, Clubs, $M, N)$ is a finite club economy in which aggregate endowment is strictly positive, then there is a feasible state $f=(y, \nu)$ and a price system $(p, q)$ such that

- all agents choose in their budget sets

- the set of agents who do not optimize in their budget set has cardinality at most

$$
N+\mid \text { Lists }_{M} \mid+K_{1} M M^{*}\left(N+\mid \text { Lists }_{M}|+| \Omega \mid\right)+K_{2}
$$

- budgets of all club types balance

The argument parallels the proof of existence of equilibrium for continuum economies given in our (1997) companion paper. In one way the present argument is a little more delicate because we must work with the convexified excess demand correspondence (rather than the excess demand correspondence) and appeal to the Shapley-Folkman theorem (instead of to the Lyapunov convexity theorem). In another way the present argument is a little 
less delicate, because we do not need to construct equilibria for a sequence of perturbed economies, adjust the membership prices, and take limits. We construct a single perturbed economy, find an "equilibrium" for this economy, and then show that this "equilibrium" is in fact an approximate equilibrium for the original economy. ${ }^{13}$ The steps are:

1 Construct a perturbed economy $\mathcal{E}^{\prime}$ by adjoining to $A$ a few agents of each external characteristic, with appropriate endowments and utility functions.

2 Identify a compact set of prices in which an "equilibrium" price will be found.

3 Construct an excess demand correspondence and a convexified excess demand correspondence.

4 Find a fixed point of the correspondence that maximizes the value of convexified excess demand.

5 Show that, at the corresponding prices, convexified excess demand for private goods is equal to 0 and convexified demand for club memberships is an element in Cons.

6 Apply the Shapley-Folkman theorem and Lemma 3.3 to find choices for each agent which are feasible in aggregate and for which most agents choose in their budget sets and most agents optimize.

7 Apply Lemma 3.3 to find choices constituting an approximate equilibrium.

\footnotetext{
${ }^{13}$ We enclose "equilibrium" in quotes because choices in the perturbed economy lie in the convexifications of demand sets, not in the demand sets themselves.
} 


\section{Proofs}

Here we collect proofs of most of the results in the text. We begin by proving the abstract result.

Proof of Lemma 3.2 For each integer $k>0$, write $\mathcal{H}_{k}=\{x \in \mathcal{H}:|x| \leq k\}$. We first establish the following claim:

Claim There is an integer $k$ such that every element of $\mathcal{H}$ dominates some nonzero element of $\mathcal{H}_{k}$. That is, for each $x \in \mathcal{H}$ there is a $y \in \mathcal{H}_{k}, y \neq 0$ such that $x \geq y$.

To prove the Claim, suppose not. Then for each integer $k$ there is an $x^{k} \in \mathcal{H}$ which does not dominate any element of $\mathcal{H}_{k}$. In particular, $x^{k} \notin \mathcal{H}_{k}$, so $\left|x^{k}\right|>k$. For each coordinate $1 \leq i \leq n$, the sequence $\left(x_{i}^{k}\right)$ is either bounded or not. If it is bounded we may use the fact that elements of $\mathcal{H}$ have nonnegative integer coordinates to extract a subsequence that is constant valued; if it is unbounded we may extract a subsequence that is strictly increasing to infinity. Applying the same reasoning to each coordinate in turn, we may extract a subsequence $\left(x^{k_{j}}\right)$ that is non-decreasing; i.e., $x^{k_{j}} \leq x^{k_{j}+1}$ for each $j$. Set $k^{*}=\left|x^{k_{1}}\right|$. Because $k_{j} \rightarrow \infty$, there is an index $j^{*}$ such that $k_{j^{*}}>k^{*}$. Because $x^{k_{j}} \geq x^{k_{1}}$ for every $j$, it follows that $x^{k_{j^{*}}} \geq x^{k_{1}}$. On the other hand, $x^{k_{1}}$ is an element of $\mathcal{H}_{k^{*}}$ which is a subset of $\mathcal{H}_{k_{j^{*}}}$, so $x^{k_{j^{*}}}$ dominates an element of $\mathcal{H}_{k_{j^{*}}}$. This is a contradiction, so we obtain the Claim.

Now let $\mathcal{G}=\mathcal{H}_{k}$; we assert that $\mathcal{G}$ generates $\mathcal{H}$. We must show that every element of $\mathcal{H}$ can be written as a non-negative integer combination of elements of $\mathcal{G}$. Note that

$$
\mathcal{H}=\bigcup_{r=1}^{\infty} \mathcal{H}_{r}
$$

Hence it suffices to show that for each $r$, every element of $\mathcal{H}_{r}$ can be written as a non-negative integer combination of elements of $\mathcal{G}$. To see this, suppose not. Then there is a smallest index $r$ such that not every element of $\mathcal{H}_{r}$ can be written as a non-negative integer combination of elements of $\mathcal{G}$. Certainly 
$r>k$ because $\mathcal{G} \supset \mathcal{H}_{k}$. Let $x \in \mathcal{H}_{r}$. By the Claim, there is a nonzero element $y \in \mathcal{G}$ with $x \geq y$. The hyotheses on $\mathcal{H}$ guarantees that $x-y \in \mathcal{H}$. Because $|x-y|<r$, minimality of $r$ entails that $x-y$ can be written as a non-negative integer combination of elements of $\mathcal{G}$. Since $x=(x-y)+y$ and $y \in \mathcal{G}$, it follows that we can also write $x$ as a non-negative integer combination of elements of $\mathcal{G}$, as desired.

We now show that, if $\nu$ is almost integer consistent for $B$ then it is exactly integer consistent for a large subset of $B$.

Proof of Lemma 3.3 The proof proceeds through several intermediate constructions and estimations. Write

$$
s=\sum_{b \in B} \nu_{b} \in \mathbf{R}^{\mathcal{M}}
$$

and

$$
t=\sum_{b \in B} \delta_{\nu_{b}} \in \mathbf{R}^{\text {Lists }_{M}}
$$

We will find a $z \in \mathcal{J}$ such that $z \leq t$ and estimate $|z-t|$. Once that is accomplished it will be easy to constuct a set $B^{\prime} \subset B$ such that $\left|B \backslash B^{\prime}\right|=$ $|z-t|$ and

$$
z=\sum_{b \in B^{\prime}} \delta_{\nu_{b}} \in \mathcal{J}
$$

The definition of $\mathcal{J}$ and the definition and linearity of the mapping $T$ entail that

$$
\sum_{b \in B^{\prime}} \nu_{b}=\sum_{b \in B^{\prime}} T\left(\delta_{\nu_{b}}\right)=T\left(\sum_{b \in B^{\prime}} \delta_{\nu_{b}}\right) \in \text { Cons }^{*}
$$

In order to construct $z$ and estimate $|z-t|$, we first estimate dist ( $s$, Cons $\left.{ }^{*}\right)$. We then construct an $x \in$ Cons $^{*}$ for which we can estimate $|x-s|$; this estimation is made easier by arranging that $x \leq s$. From this $x$ we construct a $y \in \mathcal{J}$, and estimate $|y-t|$. Using $y$ we construct the desired $z \in \mathcal{J}$ with $z \leq t$, and estimate $|z-t|$. See Figures 1 and 2 .

Step 1 We estimate dist $\left(s\right.$, Cons $\left.^{*}\right)$. To this end, choose an element $v \in$ Cons such that

$$
|s-v|=\operatorname{dist}(s, \text { Cons })
$$



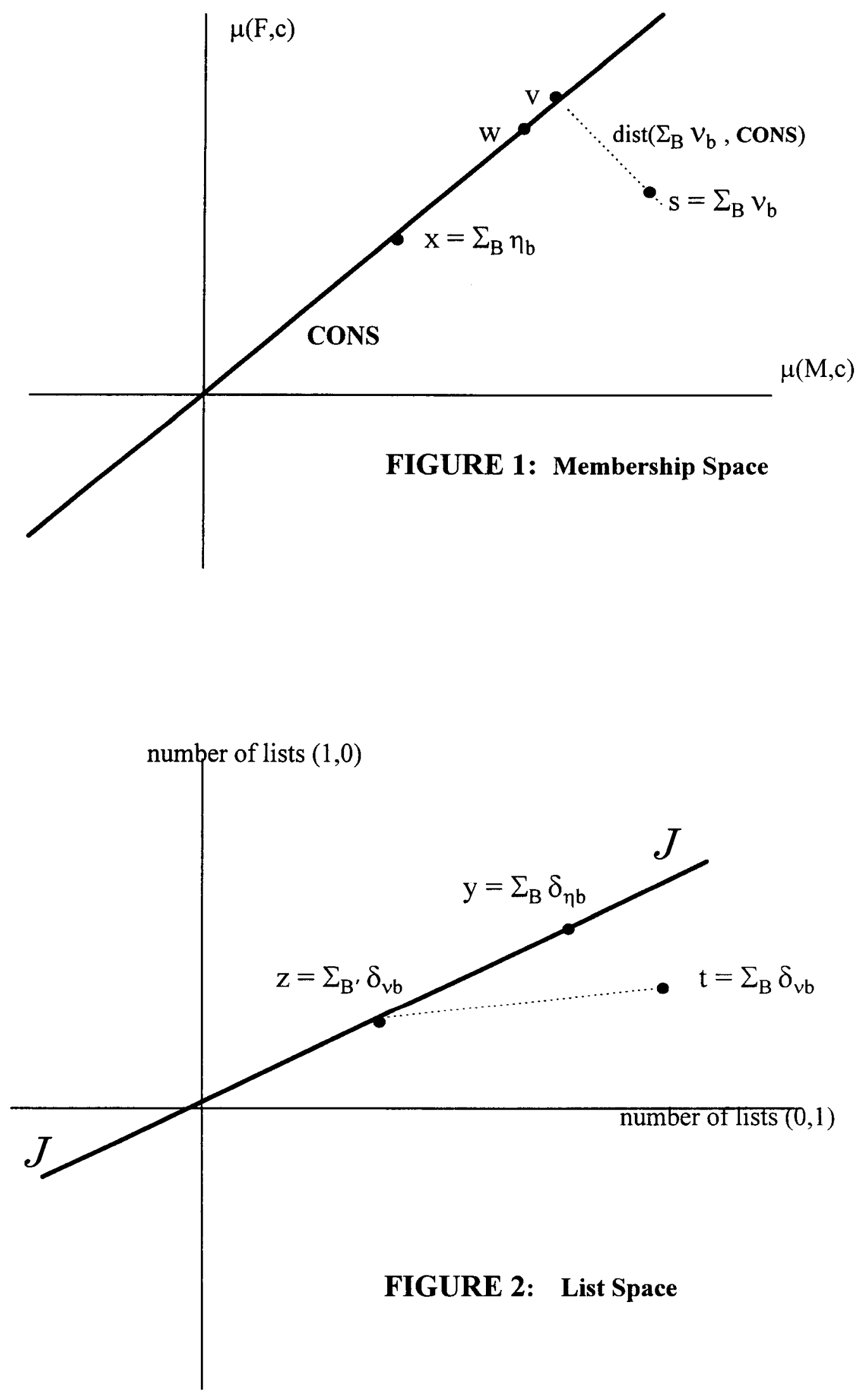
Note that $v \geq 0$, for otherwise the positive part $v^{+}$belongs to Cons (by the definition) and is closer to $s$ (which is positive) than is $v$. By definition, for each club type $(\pi, \gamma)$ there is a real number $\alpha(\pi, \gamma)$ such that for every $\omega \in \Omega$,

$$
v(\omega, \pi, \gamma)=\alpha(\pi, \gamma) \pi(\omega)
$$

Since $v \geq 0$ and $\pi \geq 0$, we may take $\alpha(\pi, \gamma) \geq 0$ for each $(\pi, \gamma)$. For each $(\pi, \gamma)$ let $\bar{\alpha}(\pi, \gamma)$ be the greatest integer less than or equal to $\alpha(\pi, \gamma)$ and let $w \in \operatorname{Lists}_{M}$ be defined by

$$
w(\omega, \pi, \gamma)=\bar{\alpha}(\pi, \gamma) \pi(\omega)
$$

for each club $(\pi, \gamma)$. This construction guarantees that $w \in$ Cons $^{*}$ and that

$$
0 \leq v(\omega, \pi, \gamma)-w(\omega, \pi, \gamma) \leq 1
$$

for each membership $(\omega, \pi, \gamma)$, so

$$
|w-v| \leq|\mathcal{M}|
$$

Hence

$$
\operatorname{dist}\left(s, \text { Cons }^{*}\right) \leq|s-w| \leq|\mathcal{M}|+\operatorname{dist}(s, \text { Cons })
$$

Step 2 We construct an element $x \in$ Cons $^{*}$ that is dominated by $s$. If $w \leq s$, take $x=w$. If $w \not z s$, there is a membership $m \in \mathcal{M}$ such that $w(m)>s(m)$. Use Lemma 3.2 to write

$$
w=\sum_{y \in \mathcal{G}} n_{w}(y) y
$$

Pick $y^{*} \in \mathcal{G}_{1}$ such that $n_{w}\left(y^{*}\right)>0$ and $y^{*}(m)>0$; set

$$
x^{1}=\left(n_{w}\left(y^{*}\right)-1\right) y^{*}+\sum_{y \neq y^{*}} n_{w}(y) y \in \text { Cons }^{*}
$$

so that $x^{1} \leq w$ and $x^{1}(m)<w(m)$. Continuing in this way we construct a decreasing sequence $x^{1} \geq x^{2} \ldots$ of elements of Cons* After at most $|s-w|$ 
iterations, we obtain a vector $x \in$ Cons $^{*}$ with $x \leq s$. Since we subtract an element of $\mathcal{G}_{1}$ at each iteration, we conclude that

$$
|w-x| \leq\left(\max _{g \in \mathcal{G}_{1}}|g|\right)|s-w|
$$

Step 3 By definition, $s=\sum b \in B \nu_{b}$ and $\nu_{b} \in \operatorname{Lists}_{M}$ for each $b$. We construct a function $\eta: B \rightarrow \operatorname{Lists}_{M}$ such that $\eta_{b} \leq \nu_{b}$ for each $b \in B$ and

$$
\sum_{b \in B} \eta_{b}=x
$$

To accomplish this, write

$$
B=\left\{b_{1}, \ldots, b_{n}\right\}
$$

Proceed inductively:

$$
\begin{aligned}
\eta_{b_{1}} & =\min \left\{\nu_{b_{1}}, x\right\} \\
\eta_{b_{2}} & =\min \left\{\nu_{b_{2}}, x-\eta_{b_{1}}\right\} \\
& \vdots \\
\eta_{b_{n}} & =x-\sum_{1 \leq i \leq n-1} \eta_{b_{i}}
\end{aligned}
$$

Step 4 Set

$$
y=\sum_{b \in B} \delta_{\eta_{b}}
$$

The definition of $T$ implies that $T(y)=x$ so $y \in \mathcal{J}$. Write

$$
B^{\prime \prime}=\left\{b \in B: \eta_{b}=\nu_{b}\right\}
$$

Because $0 \leq \eta_{b} \leq \nu_{b}$ for each $b \in B$ and $\nu_{b}-\eta_{b} \in \mathbf{Z}_{+}^{\mathcal{M}}$, it follows that

$$
\begin{aligned}
& \left|\nu_{b}-\eta_{b}\right|=0 \text { if } \quad b \in B^{\prime \prime} \\
& \left|\nu_{b}-\eta_{b}\right| \geq 1 \text { if } \quad b \in B \backslash B^{\prime \prime}
\end{aligned}
$$

Note that $\left|\delta_{\nu_{b}}-\delta_{\eta_{b}}\right|=2$ whenever $\nu_{b} \neq \eta_{b}$. Hence

$$
|y-t|=2\left|B \backslash B^{\prime \prime}\right| \text {. }
$$




\section{Moreover}

$$
|s-x|=\sum_{b \in B}\left|\nu_{b}-\eta_{b}\right|=\sum_{b \in B \backslash B^{\prime \prime}}\left|\nu_{b}-\eta_{b}\right| \geq\left|B \backslash B^{\prime \prime}\right|
$$

Step 5 Proceeding exactly as in Step 2 we construct an element $z \in \mathcal{J}$ such that $z \leq t$ and

$$
|z-y| \leq\left(\max _{g^{\prime} \in \mathcal{G}_{2}}\left|g^{\prime}\right|\right)|t-y|
$$

Step 6 For each $\ell \in$ Lists $_{M}$, write

$$
B_{\ell}=\left\{b \in B: \nu_{b}=\ell\right\}
$$

By construction, $z \leq t$ so $z(\ell) \leq t(\ell)=\left|B_{\ell}\right|$ for each $\ell$. Hence we may choose subsets $B_{\ell}^{\prime} \subset B_{\ell}$ such that $\left|B_{\ell}^{\prime}\right|=z(\ell)$. Setting

$$
B^{\prime}=\bigcup_{\ell} B_{\ell}^{\prime}
$$

therefore yields a subset $B^{\prime} \subset B$ such that

$$
\sum_{b \in B^{\prime}} \delta_{\nu_{b}}=z \in \mathcal{J}
$$

As noted at the beginning of the proof, linearity of $T$ implies

$$
\sum_{b \in B^{\prime}} \nu_{b}=\sum_{b \in B^{\prime}} T\left(\delta_{\nu_{b}}\right)=T(z) \in \text { Cons }^{*}
$$

Our construction implies that

$$
\left|B \backslash B^{\prime}\right|=\sum_{\ell \in \text { Lists }_{M}}\left|B_{\ell} \backslash B_{\ell}^{\prime}\right|=\sum_{\ell \in \text { Lists }_{M}}|t(\ell)-z(\ell)|=|t-z|
$$

Combining (1) - (6), expanding, and substituting the definitions of $K_{1}, K_{2}$ yields the required estimate for $\left|B \backslash B^{\prime}\right|$.

We can now establish our approximate decentralization result. 


\section{Proof of Theorem 3.1 Set}

$$
\begin{aligned}
N^{*} & =N+\left|\operatorname{Lists}_{M}\right| \\
K & =K_{1}\left(N^{*} M+1\right)+K_{2}+N^{*}
\end{aligned}
$$

Let $f=(x, \mu)$ be a core state. Write

$$
W=\max \left\{\left|e_{a n}\right|: a \in A, 1 \leq n \leq N\right\}
$$

Step 1 For each agent $a$, consider the preferred set

$$
\varphi(a)=\left\{(x, \ell) \in X_{a}: u_{a}(x, \ell)>u_{a}\left(x_{a}, \mu_{a}\right)\right\}
$$

and the net preferred set:

$$
\psi(a)=\left\{(x, \ell) \in \mathbf{R}^{N} \times \mathbf{R}^{\mathcal{M}}:\left(x+e_{a}-\tau(\ell), \ell\right) \in \varphi(a)\right\}
$$

Set

$$
\Psi(a)=\psi(a) \cup\{0\}
$$

Define the aggregate net preferred set

$$
Z=\sum_{a \in A} \Psi(a)
$$

\section{Step 2 Define}

$$
C^{*}=\left\{(x, \bar{\mu}) \in \mathbf{R}^{N} \times \mathbf{R}^{\mathcal{M}}: x \leq-K W 1, \operatorname{dist}(\bar{\mu}, \text { Cons }) \leq 1\right\}
$$

Note that $C^{*}$ is a convex cone. We want to separate $Z$ from $C^{*}$; to accomplish this, we must show that $C^{*} \cap \operatorname{conv} Z=\emptyset$. We suppose not and construct a blocking coalition.

Let $z^{*}=\left(x^{*}, \mu^{*}\right) \in C^{*} \cap \operatorname{conv} Z$. Note that

$$
\operatorname{conv} Z=\sum_{a \in A} \operatorname{conv} \Psi(a)
$$

Hence the Shapley-Folkman theorem guarantees that we can choose elements $\left(z_{a}, \nu_{a}\right) \in \operatorname{conv} \Psi(a)$ for each $a \in A$ in such a way that: 
(i) $z^{*}=\sum_{a \in A}\left(z_{a}, \nu_{a}\right)$

(ii) $\left|\left\{a \in A:\left(z_{a}, \nu_{a}\right) \notin \Psi(a)\right\}\right| \leq N^{*}$

Write

$$
B=\left\{a \in A:\left(z_{a}, \nu_{a}\right) \in \Psi(a)\right\}
$$

Because agents $a \in A \backslash B$ choose in the convex hull of $\Psi(a)$, and there are at most $N^{*}$ such agents, it follows that:

$$
\operatorname{dist}\left(\sum_{b \in B} \nu_{b}, \text { Cons }\right) \leq N^{*} M+\operatorname{dist}\left(\mu^{*}, \text { Cons }\right) \leq N^{*} M+1
$$

We can therefore use Lemma 3.3 to choose a subset $B^{\prime} \subset B$ such that

$$
\sum_{b \in B^{\prime}} \nu_{b} \in \text { Cons }^{*}
$$

and

$$
\left|B \backslash B^{\prime}\right| \leq K_{1}\left(N^{*} M+\operatorname{dist}\left(\mu^{*}, \text { Cons }\right)\right)+K_{2}
$$

Thus

$$
\left|A \backslash B^{\prime}\right| \leq K_{1}\left(N^{*} M+1\right)+K_{2}+N^{*}=K
$$

By assumption, $|A|>K$, so $B^{\prime} \neq \emptyset$. We assert that $B^{\prime}$ is a blocking coalition. To see this, define $y: A \rightarrow \mathbf{R}_{+}^{N}$ by

$$
y_{b}= \begin{cases}z_{b}-e_{b}+\tau\left(\nu_{b}\right) & \text { if } b \in B^{\prime} \\ 0 & \text { otherwise }\end{cases}
$$

and define the state $g=(y, \nu)$. To see that $g$ is feasible for $B^{\prime}$ note first that

$$
\left|\sum_{a \in A} e_{a}-\sum_{b \in B^{\prime}} e_{b}\right| \leq\left|A \backslash B^{\prime}\right|(W 1)
$$

Combining (7) and (8) with the fact that $\left(z^{*}, \mu^{*}\right) \in C^{*}$ yields the material balance condition. Since $\nu$ is integer consistent for $B^{\prime}$ by construction, $g$ is feasible for $B^{\prime}$. The construction of $g$ guarantees that $g(b)$ is preferred to $f(b)$ by every agent $b \in B^{\prime}$; this contradicts the core property of $f$. We conclude that $C^{*} \cap \operatorname{conv} Z=\emptyset$, as claimed. 
Step 4 We now use the separation theorem to find prices $\left(p, q^{*}\right) \in \mathbf{R}_{+}^{N} \times \mathbf{R}^{\mathcal{M}}$, $\left(p, q^{*}\right) \neq(0,0)$ and a real number $\sigma$ such that

$$
\begin{aligned}
\left(p, q^{*}\right) \cdot(x, \mu) \leq \sigma & & \text { for each }(x, \mu) \in C^{*} \\
\left(p, q^{*}\right) \cdot z \geq \sigma & & \text { for each } z \in Z
\end{aligned}
$$

Because $C^{*}$ contains a translate of $-\mathbf{R}_{+}^{N} \times\{0\}$, it follows that $p \geq 0$. Because $C^{*}$ contains a translate of $\{0\} \times$ Cons, it follows that $q^{*}$ vanishes on Cons and hence that $q^{*} \in$ Trans. We claim that $p \neq 0$. To see this, suppose to the contrary that $p=0$. By construction, $\left(p, q^{*}\right) \neq(0,0)$ so $q^{*} \neq 0$. Hence there is a $\bar{\mu} \in \mathbf{R}^{\mathcal{M}}$ such that $q^{*} \cdot \bar{\mu}>0$. For $\varepsilon>0$ sufficiently small, $(-1, \varepsilon \bar{\mu}) \in C^{*}$, so that $\left(p, q^{*}\right) \cdot(-1, \varepsilon \bar{\mu}) \leq 0$. However

$$
\begin{aligned}
\left(p, q^{*}\right) \cdot(-1, \varepsilon \bar{\mu}) & =\left(0, q^{*}\right) \cdot(-1, \varepsilon \bar{\mu}) \\
& =\varepsilon q^{*} \cdot \bar{\mu}
\end{aligned}
$$

which, by our choice of $\bar{\mu}$, is positive. This is a contradiction, so we conclude that $p \neq 0$, as asserted.

After normalizing, we may assume that $p \cdot 1=1$, so that $p \in \Delta$. Finally, because $(-K W 1,0) \in C^{*}$, it follows that

$$
\sigma \geq p \cdot(-K W 1)=-K W
$$

Define $q$ by

$$
q_{m}=q_{m}^{*}+\frac{1}{|\pi|} p \cdot \operatorname{inp}(\pi, \gamma)
$$

for each $m \in \mathcal{M}$. Note that

$$
(p, q) \cdot\left(x_{a}, \mu_{a}\right)=\left(p, q^{*}\right) \cdot\left(x_{a}+\tau\left(\mu_{a}\right), \mu_{a}\right)
$$

for each $a$.

It remains to verify the claimed estimates for $\rho^{1}, \rho^{2}$. To this end, let $E_{1} \subset A$ be the set of agents for whom $f(a)=\left(x_{a}, \mu_{a}\right)$ is not their budget 
set, and let $E_{2}=A \backslash E_{1}$. Thus, $a \in E_{1}$ if and only if expenditure strictly exceeds income:

$$
\begin{aligned}
\operatorname{expenditure}(a) & =(p, q) \cdot\left(x_{a}, \mu_{a}\right) \\
& >p \cdot e_{a}=\operatorname{income}(a)
\end{aligned}
$$

and $a \in E_{2}$ if and only if expenditure is weakly less than income:

$$
\begin{aligned}
\operatorname{expenditure}(a) & =(p, q) \cdot\left(x_{a}, \mu_{a}\right) \\
& \leq p \cdot e_{a}=\operatorname{income}(a)
\end{aligned}
$$

Because $f$ is feasible, the sum over $A$ of expenditures equals the sum over $A$ of incomes:

$$
\sum_{a \in A}(p, q) \cdot\left(x_{a}, \mu_{a}\right)=\sum_{a \in A} p \cdot e_{a}
$$

Because $A=E_{1} \cup E_{2}$, we obtain

$$
\begin{aligned}
0 & =\sum_{a \in A}\left[(p, q) \cdot\left(x_{a}, \mu_{a}\right)-p \cdot e_{a}\right] \\
& =\sum_{a \in E_{1}}\left[(p, q) \cdot\left(x_{a}, \mu_{a}\right)-p \cdot e_{a}\right]+\sum_{a \in E_{2}}\left[(p, q) \cdot\left(x_{a}, \mu_{a}\right)-p \cdot e_{a}\right]
\end{aligned}
$$

Hence

$$
\sum_{a \in E_{1}}\left[(p, q) \cdot\left(x_{a}, \mu_{a}\right)-p \cdot e_{a}\right]=-\sum_{a \in E_{2}}\left[(p, q) \cdot\left(x_{a}, \mu_{a}\right)-p \cdot e_{a}\right]
$$

By definition, $0 \in \Psi(a)$ for each $a$, and monotonicity of preferences implies that $\left(x_{a}, \mu_{a}\right)$ is in the closure of $\Psi(a)$ for each $a$. Using the separation property of prices we obtain:

$$
\sum_{a \in E_{2}}\left[(p, q) \cdot\left(x_{a}, \mu_{a}\right)-p \cdot e_{a}\right] \geq-K W
$$

Putting these together and keeping in mind that expenditure minus income is positive for agents in $E_{1}$ and no others yields:

$$
\rho^{1}(f, p, q)=\frac{1}{|A|} \sum_{a \in A} \rho_{a}^{1}(f, p, q)
$$




$$
\begin{aligned}
& =\frac{1}{|A|} \sum_{a \in A}\left[(p, q) \cdot\left(x_{a}, \mu_{a}\right)-p \cdot e_{a}\right]^{+} \\
& =\frac{1}{|A|} \sum_{a \in E_{1}}\left[(p, q) \cdot\left(x_{a}, \mu_{a}\right)-p \cdot e_{a}\right] \\
& =-\frac{1}{|A|} \sum_{a \in E_{2}}\left[(p, q) \cdot\left(x_{a}, \mu_{a}\right)-p \cdot e_{a}\right] \\
& \leq \frac{K W}{|A|}
\end{aligned}
$$

which is the desired result.

To estimate $\rho^{2}$, let $E_{3}$ be the set of agents for whom there is a choice $\left(y_{a}, v_{a}\right) \in B(a, p, q)$ that is strictly preferred to $\left(x_{a}, \mu_{a}\right)$. Just as before, separation implies

$$
\sum_{a \in E_{3}}(p, q) \cdot\left[\left(y_{a}, v_{a}\right)-\left(e_{a}, 0\right)\right] \geq-K W
$$

Rearranging yields

$$
\frac{1}{|A|} \sum_{a \in E_{3}}(p, q) \cdot\left[\left(e_{a}, 0\right)-\left(y_{a}, v_{a}\right)\right] \leq \frac{K W}{|A|}
$$

Since this inequality holds for all choices $\left(y_{a}, v_{a}\right)$, we obtain the desired inequality.

We now turn to the existence of approximate equilibrium.

\section{Proof of Theorem 4.1 Write}

$$
W=\max \left\{e_{a n}: a \in A, 1 \leq n \leq N\right\}
$$

By assumption, aggregate endowment $\bar{e}$ is strictly positive; say $\bar{e} \geq w 1>0$.

Step 1 We construct a perturbed economy $\mathcal{E}^{\prime}$ by adjoining to the agent set $A$ a single agent of each external characteristic. That is, the agent set for the perturbed economy is:

$$
A^{\prime}=A \cup\left\{a_{\omega}: \omega \in \Omega\right\}
$$


External characteristics, consumption sets, endowments and utility functions for agents in $A$ are as in the original economy $\mathcal{E}$. For agents $a_{\omega} \in A^{*}=A^{\prime} \backslash A$ we define external characteristics, consumption sets, endowments and utility functions by:

$$
\begin{aligned}
\omega_{a_{\omega}} & =\omega \\
X_{a_{\omega}} & =\mathbf{R}_{+}^{N} \times\left\{\ell \in \operatorname{Lists}_{M}: \ell\left(\omega^{\prime}, \pi, \gamma\right)=0 \text { if } \omega^{\prime} \neq \omega\right\} \\
e_{a_{\omega}} & =W 1 \\
u_{a_{\omega}}(x, \ell) & =|x|
\end{aligned}
$$

Step 2 Choose a real number $\varepsilon>0$ so small that

$$
[1-(N-1) \varepsilon]\left[\frac{W(|A|+|\Omega|)}{N \varepsilon}-W(|A|+|\Omega|)\right]-\varepsilon(N-1) W(|A|+|\Omega|)>0
$$

Having chosen $\varepsilon$, choose a real number $R>0$ so big that

$$
[1-(N-1) \varepsilon]\left[\frac{R}{N M^{*}}-W(|A|+|\Omega|)\right]-\varepsilon(N-1) W(|A|+|\Omega|)>0
$$

Define :

$$
\begin{aligned}
\Delta_{\varepsilon} & =\left\{p \in \mathbf{R}_{+}^{N}: p_{n} \geq \varepsilon \text { for each } n\right\} \\
Q_{R} & =\left\{q \in \text { Trans }:\left|q_{m}\right| \leq R \text { for all } m \in \mathcal{M}\right\}
\end{aligned}
$$

Step 3 We define an excess demand correspondence. Let $p \in \Delta_{\varepsilon}, q \in Q_{R}$. For each agent $a \in A$, write

$$
B(a, p, q)=\left\{(x, \mu) \in \mathbf{R}_{+}^{N} \times \operatorname{Lists}\left(\omega_{a}\right): p \cdot x+q \cdot \mu+p \cdot \tau(\mu) \leq p \cdot e_{a}\right\}
$$

Let

$$
\begin{aligned}
d(a, p, q)=\{(x, \mu) \in & B(a, p, q): \\
& \left.u_{a}(x, \mu) \geq u_{a}\left(x^{\prime}, \mu^{\prime}\right) \text { for all }\left(x^{\prime}, \mu^{\prime}\right) \in B(a, p, q)\right\}
\end{aligned}
$$

be agent $a$ 's demand set and let

$$
z(a, p, q)=d(a, p, q)-\left(e_{a}, 0\right)
$$


be agent $a$ 's excess demand set. Excess demand sets are uniformly bounded (because endowments are bounded, private good prices are bounded away from 0 and club membership prices are bounded above and below). Define the aggregate excess demand correspondence

$$
Z: \Delta_{\varepsilon} \times Q_{R} \rightarrow \mathbf{R}_{+}^{N} \times \mathbf{R}^{\mathcal{M}}
$$

to be the sum of the individual excess demand correspondences:

$$
Z(p, q)=\sum_{a \in A^{\prime}} z(a, p, q)
$$

Note that $Z(p, q)$ is bounded but need not be convex.

Step 4 Individual (and aggregate) excess demands for private goods lie in the compact set

$$
X=\left\{x \in \mathbf{R}^{N}:-W \leq x_{n} \leq \frac{1}{\varepsilon}(W+R M) \text { for each } n\right\}
$$

and individual and aggregate demands for club memberships lie in the set

$$
C=\left\{\bar{\mu} \in \mathbf{R}^{\mathcal{M}}: \sum_{m \in \mathcal{M}} \bar{\mu}(m) \leq M\right\}
$$

Define a correspondence

$$
\Phi: \Delta_{\varepsilon} \times Q_{R} \times X \times C \rightarrow \Delta_{\varepsilon} \times Q_{R} \times X \times C
$$

by

$\Phi(p, q, x, \mu)=\left[\operatorname{argmax}\left\{\left(p^{*}, q^{*}\right) \cdot(x, \mu):\left(p^{*}, q^{*}\right) \in \Delta_{\varepsilon} \times Q_{R}\right\}\right] \times \operatorname{conv} Z(p, q)$

It is easily checked that $\Phi$ is an upper hemi-continuous correspondence, and that its values are non-empty compact convex sets. Hence Kakutani's fixed point theorem guarantees that $\Phi$ has a fixed point. Thus there is a price pair $(p, q) \in \Delta_{\varepsilon} \times Q_{R}$ and a consumption/club membership pair $(\bar{z}, \bar{\mu}) \in$ conv $Z(p, q)$ such that

$(p, q) \cdot(\bar{z}, \bar{\mu})=\max \left\{\left(p^{*}, q^{*}\right) \cdot\left(z^{*}, \mu^{*}\right):\left(p^{*}, q^{*}\right) \in \Delta_{\varepsilon} \times Q_{R},\left(z^{*}, \mu^{*}\right) \in Z(p, q)\right\}$ 
Step 5 We show that $\bar{z}=0$ and $\bar{\mu} \in$ Cons. The argument is in several parts. Keep in mind throughout that

$$
(\bar{z}, \bar{\mu})=\sum_{a \in A^{\prime}}\left\{\left(x_{a}, \mu_{a}\right)-\left(e_{a}^{*}, 0\right)\right\}
$$

where, for each $a$, the choice $\left(x, \mu_{a}\right)$ is a convex combination of demands optimal choices at prices $(p, q)$.

Step 5.1 We show first that

$$
q \cdot \bar{\mu}=0
$$

. Suppose that this is not so; we obtain a contradiction by looking at excess demands (at prices $p, q$ ) of agents in $A^{*}=A^{\prime} \backslash A$. Maximality and the definition of $\Phi$ entail that $q \cdot \bar{\mu}>0$ (because $0 \cdot \bar{\mu}=0$ ). Maximality entails that $q \in \operatorname{bdy} Q_{R}$ so that $\left|q_{m}^{k}\right|=R$ for some $m \in \mathcal{M}$. The budget balance condition for clubs means that if some price has large magnitude and is positive then some other price must have large magnitude and be negative. Thus there is a membership $m^{*}=\left(\omega^{*}, \pi^{*}, \gamma^{*}\right)$ such that $q_{m^{*}} \leq-R / M^{*}$. The agents $a_{\omega^{*}} \in A^{\prime}$ whom we have adjoined to the original set of agents, and whose external characteristic is $\omega^{*}$, could obtain a subsidy of $R / M^{*}$ by choosing the membership $m^{*}$ (and no other). Because this agent does not care at all about club memberships and finds all private goods to be perfect substitutes, it follows that his (convexified) excess demand for one of the least expensive private goods - which we may as well suppose to be good 1 - is at least

$$
\zeta_{1}(b, p, q) \geq \frac{R}{N M^{*}}
$$

Keeping in mind that the (convexified) excess demand of every agent in $A^{\prime}$ is bounded below by $-W 1$ and that the number of agents in $A^{\prime}$ is $|A|+|\Omega|$, the aggregate (convexified) excess demand for good 1 and for other private goods satisfy:

$$
\begin{aligned}
& \bar{z}_{1} \geq \frac{R}{N M^{*}}-W(|A|+|\Omega|) \\
& \bar{z}_{n} \geq-W(|A|+|\Omega|)
\end{aligned}
$$


Define $p \in \Delta_{\varepsilon}$ by:

$$
p_{n}=\left\{\begin{array}{lll}
1-(N-1) \varepsilon & \text { if } & n=1 \\
\varepsilon & \text { if } & n>1
\end{array}\right.
$$

Calculation shows that

$$
p \cdot \bar{z} \geq[1-(N-1) \varepsilon]\left[\frac{R}{N M^{*}}-W(|A|+|\Omega|)\right]-\varepsilon(N-1) W(|A|+|\Omega|)
$$

Our choices of $R, \varepsilon$ guarantee that this is strictly positive so

$$
(p, 0) \cdot(\bar{z}, \bar{\mu})>0=(p, q) \cdot(\bar{z}, \bar{\mu})
$$

which contradicts maximality. We conclude that $q \cdot \bar{\mu}=0$, as desired.

Step 5.2 We show next that $\bar{\mu} \in$ Cons. If not, we could find a pure transfer $q^{*} \in$ Trans such that $q^{*} \cdot \bar{\mu}>0$ and hence could find a $q^{* *} \in Q_{R}$ such that $q^{* *} \cdot \bar{\mu}>0$, contradicting maximality.

Step 5.3 We claim that $p_{n}>\varepsilon$ for each $n$. Suppose not; we once again obtain a contradiction by considering the excess demand of agents in $A^{*}=A^{\prime} \backslash A$. Every agent in $A^{*}$ finds all commodities to be perfect substitutes, and therefore demands only the least expensive commodities. Because agents in $A^{*}$ have endowment $W 1$ and hence wealth $W$, there is at least one commodity, say commodity 1 , for which the excess demand of each agent in $A^{*}$ is at least

$$
\zeta_{1}(a, p, q) \geq \frac{W}{N \varepsilon}
$$

Summing over all agents and keeping in mind that individual excess demands are bounded below by $-W 1$, we conclude that

$$
\begin{aligned}
& \bar{z}_{1} \geq \frac{W(|A|+|\Omega|)}{N \varepsilon}-W(|A|+|\Omega|) \\
& \bar{z}_{n} \geq-W(|A|+|\Omega|)
\end{aligned}
$$

Define $p \in \Delta_{\varepsilon}$ by

$$
p_{n}=\left\{\begin{array}{lll}
1-(N-1) \varepsilon & \text { if } & n=1 \\
\varepsilon & \text { if } & n>1
\end{array}\right.
$$


Calculation gives

$p \cdot \bar{z} \geq[1-(N-1) \varepsilon]\left[\frac{W(|A|+|\Omega|)}{N \varepsilon}-W(|A|+|\Omega|)\right]-\varepsilon(N-1) W(|A|+|\Omega|)$

Our choice of $\varepsilon$ guarantees that this is strictly positive and hence that

$$
(p, 0) \cdot(\bar{z}, \bar{\mu})>0=(p, q) \cdot(\bar{z}, \bar{\mu})
$$

which again contradicts maximality. We conclude that $p_{n}>\varepsilon$ for each $n$.

Step 5.4 We show that $\bar{z}=0$. If $\bar{z} \neq 0$ there are indices $i, j$ such that $\bar{z}_{i}<0$ and $\bar{z}_{j}>0$. Since $\left.(p, q) \cdot \bar{z}, \bar{\mu}\right)=0$ and $q \cdot \bar{\mu}=0$ it follows that $p \cdot \bar{z}=0$. Since $p_{i}>\varepsilon$, we can construct a price $\hat{p} \in \Delta_{\varepsilon}$ by setting

$$
\hat{p}_{n}= \begin{cases}p_{i}-\frac{1}{2}\left(p_{i}-\varepsilon\right) & \text { if } n=i \\ p_{j}+\frac{1}{2}\left(p_{i}-\varepsilon\right) & \text { if } n=j \\ p_{n} & \text { otherwise }\end{cases}
$$

Since $p \cdot \bar{z}=0$, it follows that $\hat{p} \cdot \bar{z}>0$, a contradiction. We conclude that $\bar{z}=0$.

Step 6 Applying the Shapley-Folkman theorem, we find choices $\left(x_{a}, \mu_{a}\right) \in$ conv $d(a, p, q)$ and a set $A^{\prime \prime} \subset A^{\prime}$ such that

- $\sum_{a \in A^{\prime}}\left(x_{a}, \mu_{a}\right)=(\bar{z}, \bar{\mu})$

- $\left(x_{a}, \mu_{a}\right) \in d(a, p, q)$ if $a \in A^{\prime \prime}$

- $\left|A^{\prime} \backslash A^{\prime \prime}\right| \leq N+\mid$ Lists $_{M} \mid$

Set $B=A \cap A^{\prime \prime} ;$ note that

$$
|A \backslash B| \leq N+\left|\operatorname{Lists}_{M}\right|
$$

and

$$
\left|A^{\prime} \backslash B\right| \leq N+\mid \text { ists }_{M}|+| \Omega \mid
$$


By construction,

$$
\sum_{a \in A^{\prime}} \mu_{a}=\bar{\mu} \in \text { Cons }^{*}
$$

so

$$
\operatorname{dist}\left(\sum_{a \in B} \mu_{a}, \text { Cons }^{*}\right) \leq M M^{*}\left(N+\left|\operatorname{Lists}_{M}\right|+|\Omega|\right)
$$

Apply Lemma 3.3 to find a subset $B^{\prime} \subset B$ such that

$$
\sum_{a \in B} \mu_{a} \in \text { Cons* }^{*}
$$

and

$$
\left|B \backslash B^{\prime}\right| \leq K_{1} \operatorname{dist}\left(\sum_{a \in B} \mu_{a}, \text { Cons }^{*}\right)+K_{2}
$$

Define a state $f=\left(y_{a}, \nu_{a}\right)$ by

$$
\left(y_{a}, \nu_{a}\right)=\left\{\begin{array}{lll}
\left(x_{a}, \nu_{a}\right) & \text { if } & a \in B^{\prime} \\
(0,0) & \text { if } & a \notin B^{\prime}
\end{array}\right.
$$

The state $f$ is feasible for $A$ and has the property that all agents choose in their budget sets and agents in $B^{\prime}$ optimize. Combining equations (9), (10) and (11) yields the desired estimate on the cardinality of the set of agents who do not optimize in their budget sets. 


\section{References}

[1] R. M. Anderson, "An Elementary Core Equivalence Theorem", Econometrica 46 (1978), 1483-1487.

[2] E. Berglas, "On the Distribution of Tastes and Skills in the Provision of Local Public Goods," Journal of Public Economics 6 (1976), 409-423.

[3] E. Berglas, "On the Theory of Clubs," American Economic Review 66 (1981), 116-121.

[4] E. Berglas and D. Pines, "Clubs as a Case of Competitive Industry with Goods of Variable Quality," Economics Letters 5 (1980), 363-366.

[5] E. Berglas and D. Pines, "Clubs, Local Public Goods, and Transportation Models: A Synthesis," Journal of Public Economics 15 (1981), $141-162$.

[6] T. Bewley, "A Critique of Tiebout's Theory of Local Public Expenditures," Econometrica 49 (1981), 713-740.

[7] R. Boadway, "On the Method of Taxation and the Provision of Local Public Goods: Comment," American Economic Review 72 (1982), 846851.

[8] J. Brueckner, "Tastes, Skills and Local Public Goods," Journal of Urban Economics 35 (1994), 201-220.

[9] J. Buchanan, “An Economic Theory of Clubs," Economica 33 (1965), 1-14.

[10] H. Cole and E. Prescott, "Valuation Equilibrium with Clubs," Staff Report 174, Research Department, Federal Reserve Bank of Minneapolis, Minnesota (1994).

[11] J. P. Conley and M. Wooders, "Equivalence of the Core and Competitive Equilibrium in a Tiebout Economy with Taste Types," Working 
Paper, Department of Economics, University of Illinois, Champaign (1994).

[12] R. Cornes and T. Sandler, The Theory of Externalities, Public Goods and Club Goods, Cambridge University Press, Cambridge (1986).

[13] D. Diamantaras and R.P. Gilles, "The Pure Theory of Public Goods: Efficiency, Decentralization and the Core," International Economic Review 37 (1996), 851-860.

[14] D. Diamantaras, R. P. Gilles and S. Scotchmer, "Decentralization of Pareto Optima in Economies with Public Projects and Nonessential Private Goods," Economic Theory 8 (1996), 555-564.

[15] B. Ellickson, "A Generalization of the Pure Theory of Public Goods," American Economic Review 63 (1973), 417-432.

[16] B. Ellickson, "Competitive Equilibrium with Local Public Goods," Journal of Economic Theory 21 (1979), 46-61.

[17] B. Ellickson, B. Grodal, S. Scotchmer and W. Zame, "Clubs and the Market: Continuum Economies," UCLA Working Paper (1997).

[18] G. Engl and S. Scotchmer, "The Core and the Hedonic Core: Equivalence and Comparative Statics," Journal of Mathematical Economics 26 (1996), 209-248.

[19] G. Engl and S. Scotchmer, "The Law of Supply in Games, Markets and Matching Models," Economic Theory (forthcoming).

[20] R. P. Gilles and S. Scotchmer, "Decentralization in Replicated Club Economies with Multiple Private Goods," Journal of Economic Theory 72 (1997), 363-387.

[21] J. Greenberg and B. Shitovitz, "Consistent Voting Rules for Competitive Local Public Goods Economies," Journal of Economic Theory 46 (1988), 223-236. 
[22] J. Greenberg and S. Weber, "Strong Tiebout Equilibrium under Restricted Preferences Domain," Journal of Economic Theory 38 (1986), 101-117.

[23] A. Mas-Colell, "Efficiency and Decentralization in the Pure Theory of Public Goods," Quarterly Journal of Economics 94 (1980), 625-641.

[24] M. V. Pauly, "Clubs, Commonality and the Core: An Integration of Game Theory and the Theory of Public Goods," Economica 34 (1967), 314-324.

[25] M. V. Pauly, "Cores and Clubs," Public Choice 9 (1970), 53-65.

[26] D. Pines, "Tiebout without Politics," Regional Science and Urban Economics 21 (1991), 469-489.

[27] S. Scotchmer, "Profit-Maximizing Clubs," Journal of Public Economics 27 (1985), 25-45.

[28] S. Scotchmer, "Two-Tier Pricing of Shared Facilities in a Free-Entry Equilibrium," Rand Journal of Economics 16 (1985), 456-472.

[29] S. Scotchmer, "Public Goods and the Invisible Hand," in J. Quigley and E. Smolensky (eds.), Modern Public Finance, Harvard University Press, Cambridge (1994).

[30] S. Scotchmer, "Externality Pricing in Club Economies," Ricerche Economiche 50 (1996), 347-366.

[31] S. Scotchmer, "On Price-Taking Equilibrium in Club Economies with Nonanonymous Crowding," Journal of Public Economics, forthcoming (1997).

[32] S. Scotchmer and M. Wooders, "Competitive Equilibrium and the Core in Club Economies with Anonymous Crowding," Journal of Public Economics 34 (1987), 159-174. 
[33] C. M. Tiebout, "A Pure Theory of Local Public Goods," Journal of Political Economy 64 (1956), 416-424.

[34] M. H. Wooders, "Equilibria, the Core and Jurisdiction Structures in Economies with a Local Public Good," Journal of Economic Theory 18 (1978), 328-348, and "Correction," Journal of Economic Theory 25 (1981), 144-151.

[35] M. H. Wooders, "A Tiebout Theorem," Mathematical Social Sciences 18 (1989), 33-55. 